\title{
Opioid-Induced Constipation in Real-World Practice: A Physician Survey, 1 Year Later
}

\author{
Domenico Alvaro (D) - Flaminia Coluzzi (D) - Walter Gianni • \\ Fabio Lugoboni (D) - Franco Marinangeli (D) - Giuseppe Massazza • \\ Carmine Pinto (D) - Giustino Varrassi (D)
}

Received: November 26, 2021 / Accepted: January 14, 2022 / Published online: February 5, 2022

(C) The Author(s) 2022

\begin{abstract}
Introduction: Opioid-induced constipation (OIC) is the most common adverse effect of opioid therapy, but it is underdiagnosed and undertreated. Last year, a survey among Italian healthcare providers revealed important differences in the clinical management of OIC across physician specialties, the need of
\end{abstract}

All authors contributed equally to the manuscript and are therefore listed alphabetically.

Supplementary Information The online version contains supplementary material available at https:// doi.org/10.1007/s40122-022-00354-4.

\section{Alvaro}

Department of Translational and Precision

Medicine, Gastroenterology Division, Sapienza

University of Rome, Rome, RM, Italy

F. Coluzzi $(\bowtie)$

Department of Medical and Surgical Sciences and Biotechnologies, SAPIENZA University of Rome, Polo Pontino, Latina, Corso della Repubblica 79, 04100 Latina, LT, Italy

e-mail: flaminia.coluzzi@uniroma1.it

F. Coluzzi

Unit of Anesthesia, Intensive Care and Pain

Medicine, Sant'Andrea University Hospital, Rome,

RM, Italy

W. Gianni

Department of Internal Medicine and Geriatry, University Hospital Policlinico Umberto I, Rome, RM, Italy standardization of diagnosis and treatment, and the urgency of further education. Herein, we submitted an updated version of the survey to the same cohort of experts to evaluate potential progress.

Methods: The online survey included 15 questions about OIC. Responses were analyzed descriptively and aggregated by physician specialty.

Results: A total of 190 physicians completed the survey. Most respondents (65\%) did not feel adequately educated about OIC despite general consensus regarding interest in the topic and acknowledgement of OIC impact on patients' QoL and adherence to opioid therapy. Overall,

\section{F. Lugoboni}

Department of Medicine, Addiction Unit, University Hospital of Verona, Verona, VR, Italy

\section{F. Marinangeli}

Department of Anesthesiology, Pain Medicine, and Palliative Care, University of L'Aquila, L'Aquila, AQ, Italy

\section{G. Massazza}

Division of Physical Medicine and Rehabilitation, Department of Surgical Sciences, University of Turin and "Città della Salute e della Scienza" University Hospital, Turin, TO, Italy

C. Pinto

Medical Oncology Unit, Clinical Cancer Center, AUSL-IRCCS of Reggio Emilia, Reggio Emilia, RE, Italy

G. Varrassi

Paolo Procacci Foundation, Rome, RM, Italy 
$55-77 \%$ of physicians regularly evaluated intestinal function or OIC symptoms in patients receiving opioid therapy, with one-third of respondents implementing it in the past year. Even though the most common method for assessment was still patient diary, the use of specific scales underwent a small but significant increase compared to the previous year, with major implementation in the use of Rome IV criteria. As regards first-line treatment, most respondents (49\%) preferred macrogol prophylaxis followed by macrogol plus another laxative. For second-line treatment, we revealed a growth in the prescription of peripherally acting mu-opioid receptor antagonists (PAMORAs), with $46 \%$ of all the respondents having increased their use during the past year. Conclusions: Despite some limitations, our study demonstrated a slow but important step closer to standardization of diagnosis and treatment of OIC. Further educational and training efforts should be put in place to favor best evidence-based clinical practice.

Keywords: Chronic pain; Opioid; Opioidinduced constipation; PAMORAs

\section{Key Summary Points}

Opioid-induced constipation (OIC) is the most common adverse effect of opioid therapy.

A 2020 survey showed variability in the management of OIC across Italian specialists and the need of standardization of diagnosis and therapy.

An updated version of the survey was performed in 2021 to report potential progress.

We found an implementation in the evaluation of the intestinal function and in the prescription of recommended second-line treatments.

However, there is still a need for further education and for a more homogeneous use of specific diagnostic scales.

\section{INTRODUCTION}

Despite that opioid therapy is a valuable and effective tool in achieving optimal control of acute and chronic pain, it is associated with many adverse reactions, the most prevalent of which is opioid-induced constipation (OIC) $[1,2]$. OIC consistently affects activities of daily living, patient health-related quality of life (QoL), and adherence to therapy [3-5]. It occurs in $51-87 \%$ of patients with cancer pain [6-8], $41-57 \%$ of patients with chronic non-cancer pain [3], and an estimated 56\% of patients receiving substitution therapy for opioid dependence [9].

OIC results from the activation of mu-opioid receptors in the myenteric and submucosal plexus of the enteric nervous system, affecting gastrointestinal motility, secretion, and sphincter function [10].

Aside from dietary changes and lifestyle modifications, the most recent recommendations for the pharmacological management of OIC identify conventional laxatives as first-line treatment [7, 11-15], serotoninergic neuroenteric modulators (e.g., prucalopride), and peripherally acting mu-opioid receptor antagonists (PAMORAs) for second-line treatment [16]. PAMORAs are designed to antagonize the effects of opioids in the gastrointestinal system without affecting their central analgesic benefits. In the nation-wide survey we carried out in 2020 among Italian specialists, it emerged that although most physicians followed guideline recommendations for first-line therapy, few of them have ever prescribed PAMORAs [17]. At least in part, this may depend on the fact that PAMORA use in Italy was limited by nota 90 , a regulation that only allowed their prescription through the national health system (NHS) for OIC in terminal patients. However, on April, 30 2020, nota 90 was modified to allow the prescription of PAMORAs to all patients with OIC under the Italian NHS, thus reducing the costprohibitive nature of these medications and possibly facilitating their prescription as a second-line therapy for OIC.

In the aforementioned survey, we examined common practices and perceptions about OIC 
among Italian physicians who prescribe opioids [17]. We detected a general interest in improving the standard of care for OIC patients, but we also observed a lack of adequate education about best practices in diagnosis, prophylaxis, and treatment. In addition, another survey recently carried out in Italy and involving opioid-treated patients documented general dissatisfaction with OIC management and a definite need for better education of healthcare providers about this condition [5].

These results, together with the recent modification of nota 90 , should aid to close the gap between current knowledge about OIC and real-world clinical practice. In order to evaluate potential progress and additional educational needs regarding this topic, an updated version of the survey was administered to the same cohort of Italian physicians 1 year later.

\section{METHODS}

\section{Study Design}

This study is an updated version of a nationwide survey administered to a cohort of 501 physicians who answered a previous survey regarding OIC management in Italy in 2020 [17].

The survey was active for a period of around 8 weeks, from the beginning of June 2021 to the end of July 2021. It was e-mailed using a password-protected web link to 501 specialistsanesthesiologists/pain therapists, oncologists, gastroenterologists, geriatricians, psychiatrists/ addiction medicine specialists, general practitioners, internal medicine specialists, orthopedists, rehabilitation therapists, and palliative care specialists-involved in the management of patients under opioid therapy, comprising those with cancer and non-cancer pain as well as those on opioid substitution therapy.

Survey questions were based on those of the original survey and were slightly modified and implemented by a multidisciplinary scientific board on the basis of physician experience, available literature and current guidelines (see Table 1 for survey questions and 'Supplementary Material' for the complete survey). The board was comprised of eight experts in the fields of oncology, orthopedic surgery and rehabilitative medicine, anesthesiology and pain medicine, gerontology, gastroenterology, palliative care, and psychiatry.

Survey responses were anonymized and handled via remote dispersed geographic participation. Like the previous inquiry [17], even the updated survey was not subject to approval by an ethical committee as per Italian law and international guidelines. All the physicians involved in the survey consented to have their responses used in a medical publication.

\section{Survey}

The survey included 15 questions about common practices and perception regarding OIC in the following fields: awareness and education; diagnosis; prevention and treatment; perceived effect of OIC on patient QoL; treatment modification; patient information. Additional questions about changes occurred during the past year were present (See Table 1 and 'Supplementary Material' for survey questions and the complete survey, respectively). Question responses were multiple-choice and either single response or multiple response depending on the type of the question. Survey responses were tabulated by medical specialty or aggregated by physician specialty category and descriptively summarized as the number and percentage.

\section{RESULTS}

\section{Characteristics of Respondents}

The survey was e-mailed to the 501 Italian physicians who answered the original survey about OIC in 2020 [17]; of these, 225 (45\%) accepted the invitation. Thirty-five respondents provided only partial responses. Of the 225 respondents who started the survey, $25.8 \%$ were general practitioners (58 respondents), 18.7\% (42 respondents) were psychiatrists, 13.8\% (31 respondents) were anesthesiologists/pain therapists, $8.9 \%$ (20 respondents) were oncologists, $8 \%$ (18 respondents) were palliative care specialists, $\quad 5.8 \% \quad$ (13 respondents) were 
Table 1 Survey questions

\section{Number Survey questions}

Q1A In your clinical practice, do you regularly evaluate pain?

Q1B In the past year, have you modified your clinical practice regarding pain assessment?

Q2A At the center where you practice medicine, is intestinal function evaluated regularly? If regularly, how frequently?

Q2B In the past year, has the center where you practice medicine implemented the assessment of intestinal function in patients?

Q3 In patients who chronically use opioids, do you systematically evaluate intestinal function?

Q4A What criteria do you use for constipation assessment (multiple responses allowed)?

Q4B In the past year, did you use the diagnostic criteria for constipation assessment (Rome IV) more frequently?

Q5A In clinical practice, in a patient receiving treatment with an opioid agonist, information about the possible emergence of constipation symptoms and indications for its prevention/management:

Q5B In the past year, have you provided more information to opioid-treated patients regarding the onset of potential constipation and its prevention/management?

Q6 How many cases of opioid-induced constipation do you observe in your clinical practice?

Q7 Other than dietary and lifestyle measures, what first-line therapy do you find to be useful for the management of opioid-induced constipation?

Q8 In patients receiving laxative treatment for opioid-induced constipation, in what percentage do you prescribe a PAMORA (peripherally acting mu-opioid receptor antagonist)?

Q9 In the past year did you modify your clinical practice in patients treated for OIC, increasing the use of PAMORA?

Q10 In your opinion, to what degree the lack of OIC management influence the quality of life of your patients?

Q11A Do you agree that opioid-induced constipation can negatively influence adherence to analgesic therapy or opioid substitution therapy?

Q11B If you answered "yes" to question 11A, please indicate the type of modifications that were applied to ongoing analgesic therapy

Q12A Do you treat OIC differently when exacerbants are present?

Q12B If you answered “yes” to question 12A, which of the following factors do you evaluate?

Q13A In case of OIC which kind of treatment do you use?

Q13B In case of opioid-exacerbated constipation (OEC) which kind of treatment do you use?

Q14 Express your interest in the topic of opioid-induced constipation on a scale from 0 (no interest) to 10 (maximum interest)

Q15 Do you feel adequately educated about the treatment of opioid-induced constipation?

rehabilitation medicine specialists, $5.3 \% \quad(12$ respondents) were orthopedists, $4.9 \% \quad(11$ respondents) were internal medicine specialists. Other physician categories (gastroenterology, gerontology, and addiction medicine) had less than ten respondents each.

The majority of respondents (84\%) regularly evaluated pain among their patients, either 
using appropriate scales (38\%) or directly asking the patient/caregiver if she/he was experiencing it $(46 \%)(\mathrm{Q} 1 \mathrm{~A})$, whereas $11 \%$ of respondents left pain assessment and treatment to a pain specialist or a nurse. During the past year, onethird of respondents (33\%) changed their routine pain assessment, implementing it (Q1B), while most of the others (48\%) were already evaluating pain in their clinical practice.

\section{Awareness and Education on OIC}

Similar to the previous survey [17], the majority of respondents $(54 \%)$ expressed high interest (score of $8-10$ on a $0-10$ numerical rating scale) in the topic of OIC; $40 \%$ indicated a medium level of interest (score 5-7); and 6\% indicated low or no interest (score 0-4) (Q14). Confirming our previous data, the level of interest was not homogeneous across physician specialties with highest levels for anesthesiologists/pain therapists and low-to-moderate levels for most of psychiatrists.

Thirty-two percent of respondents felt satisfied with their current level of education regarding OIC, whereas 59\% did not feel adequately educated about it, though they were eager to learn more. Conversely, 9\% of respondents were neither sufficiently informed nor interested in additional education/training on OIC, a percentage that is slightly increased in comparison with the previous survey (Q15) (Fig. 1).

Fifty-two percent of physicians indicated that they saw less than five cases of OIC per month in clinical practice, $11 \%$ less than the

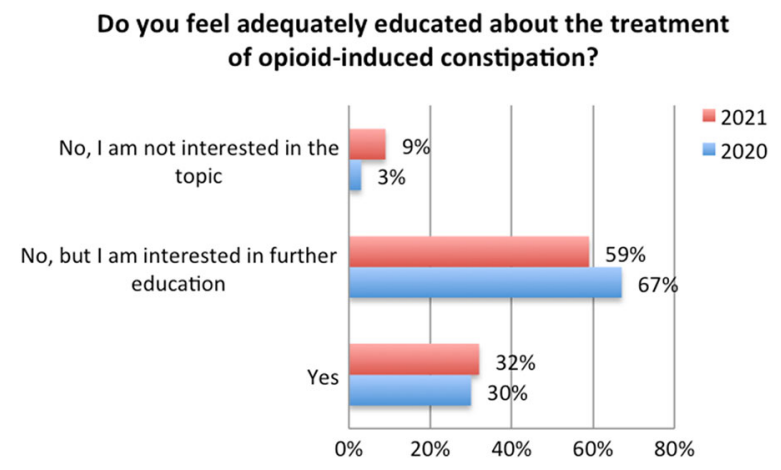

Fig. 1 OIC education previous year (Q6). Only 5\% of respondents observed more than 20 cases per month, whereas $11 \%$ saw no case at all.

\section{Diagnosis of OIC}

In general, most respondents reported that they evaluated the intestinal function of their patients at every visit (35\%) or at regular intervals $(20 \%)$, at their respective centers (Q2A) (Fig. 2A). A quarter of the total declared to assess it only occasionally, while $18 \%$ evaluated intestinal function only in case the patient/caregiver reported a gastrointestinal (GI) disturbance.

During the past year, approximately onethird of respondents $(32 \%)$ reported that the center where they practice medicine implemented the assessment of intestinal function in patients (Q2B) (Fig. 2B), with the highest degree of implementation among internal medicine doctors (45\%) and anesthesiologists/pain therapists (44\%).

Consistent with these general results and confirming our previous data, $77 \%$ of respondents indicated that intestinal function was regularly evaluated in patients on long-term opioid therapy (Q3). Of these, 22\% used a specific scale or questionnaire to assess constipation, whereas the remaining 55\% relied on the patient/caregiver to know if the patient defecated regularly. Though still low, the proportion of specialists using specific scales almost doubled compared to 2020 .

The most frequently used method for constipation assessment was still represented by patient/caregiver diaries (36\%), even though reduced compared to the previous year (53\%) (Q4A). The use of specific scales ranged from 6 to $15 \%$ of participants, with the Bowel Function Index (BFI) and the Bristol Stool Scale (BSS) used in $6 \%$ and $11 \%$ of cases, respectively, and Rome IV criteria utilized in $15 \%$ of cases (Fig. 3A). However, it is noteworthy that $31 \%$ of respondents did not use any method to assess OIC, in particular among psychiatrists.

The majority of surveyed physicians (45\%) reported that during the past year they diagnosed constipation with criteria other than 


\section{A At the center where you practice medicine, is intestinal} function evaluated regularly?

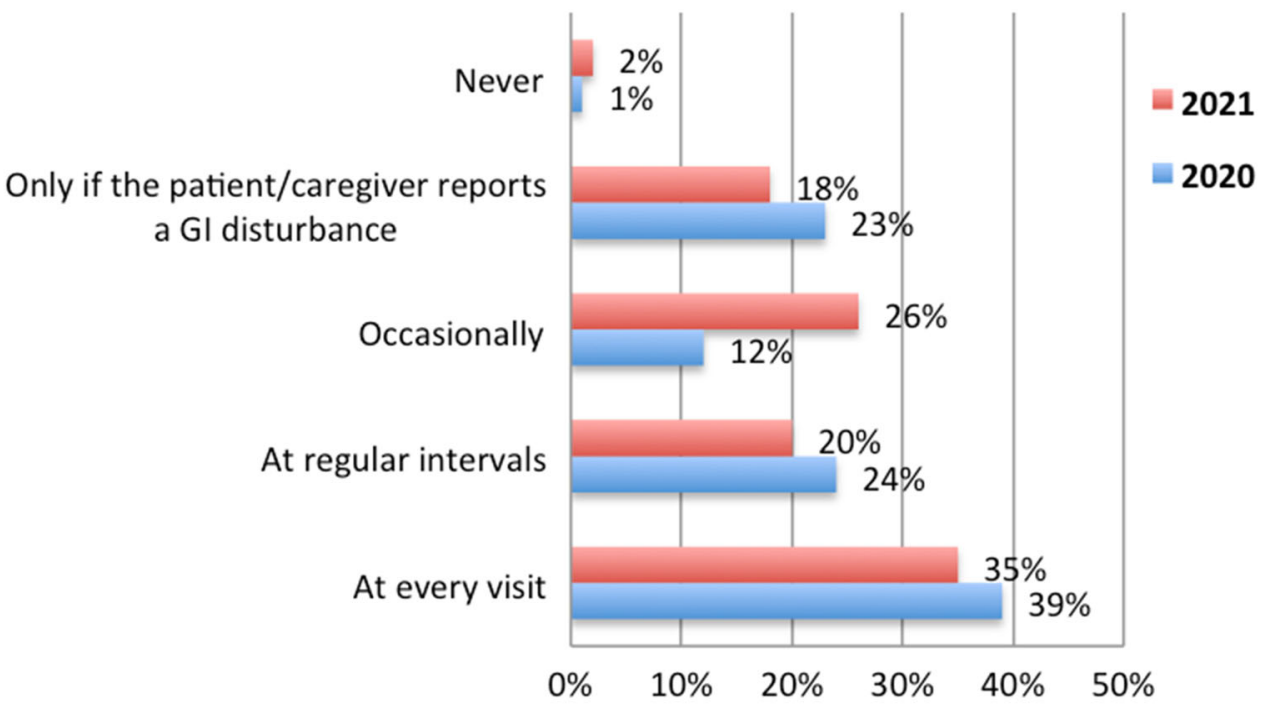

B In the past year, has the center where you practice medicine implemented the assessment of intestinal function in patients?

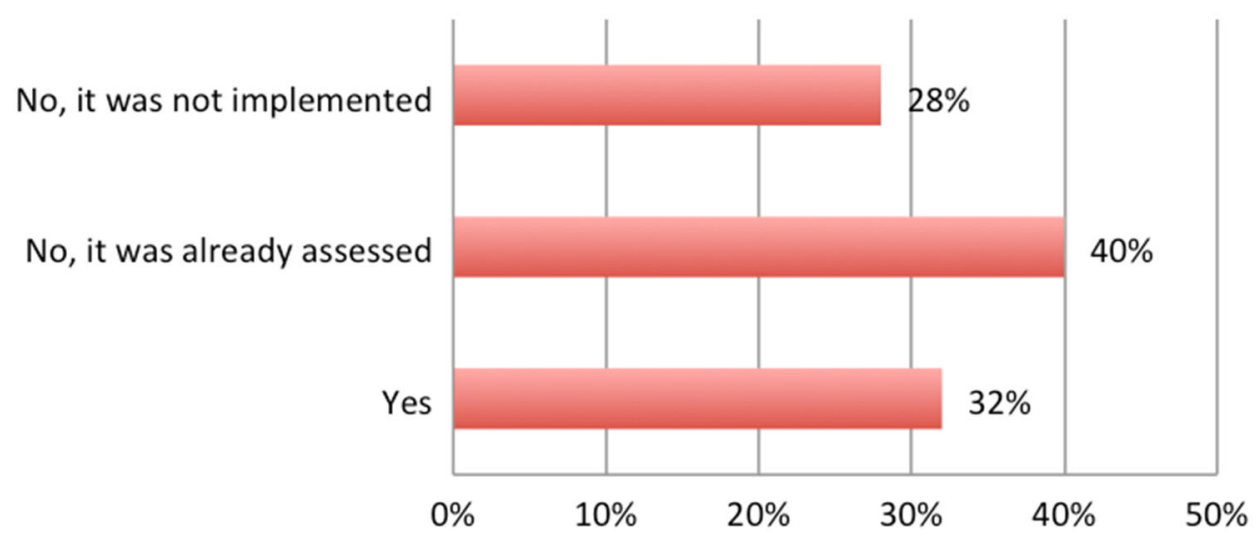

Fig. 2 OIC evaluation. A Evaluation and $\mathbf{B}$ implementation of intestinal function

Rome IV, but some of them implemented their use (13\%), utilized them in some patients $(18 \%)$ or were already using them (20\%) (Q4B) (Fig. 3B). The highest implementation in the use of Rome IV was seen among palliative care specialists $(28 \%)$, followed by anesthesiologists/pain therapists (20\%). Instead, $58 \%$ of psychiatrists indicated that they generally use other methodologies to evaluate constipation.

\section{OIC Prevention and Treatment}

Distinction of OIC from constipation exacerbated by opioid use or opioid-exacerbated constipation (OEC) is always important, particularly for the purpose of selecting an appropriate treatment. Consistently, the majority of respondents (62\%) treated OIC differently when exacerbating factors were present (Q12A). Like in the previous inquiry, almost all 
A What criteria do you use for constipation assessment?
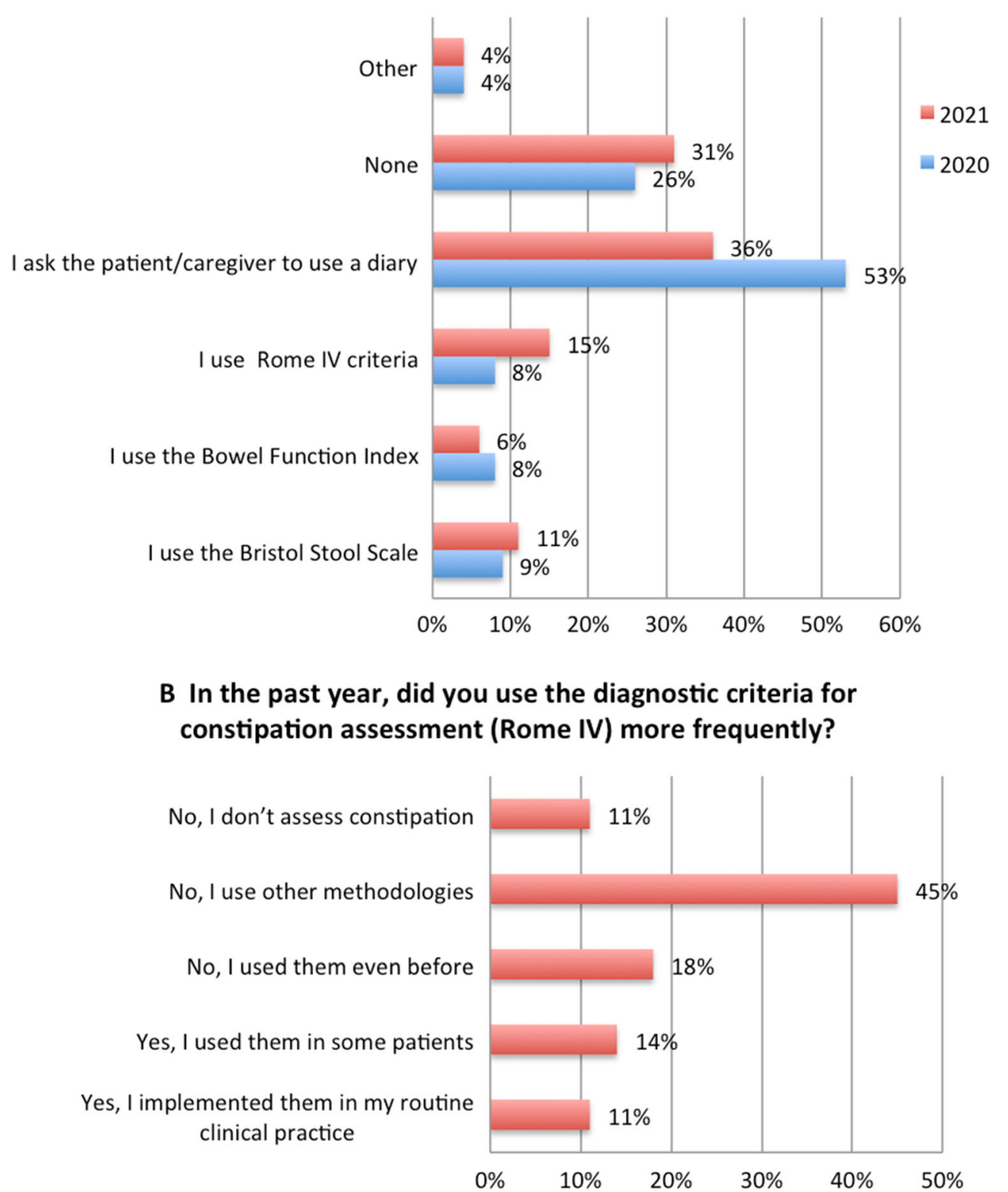

Fig. 3 OIC assessment. A Criteria for constipation assessment and $\mathbf{B}$ their implementation

the surveyed physicians evaluated exacerbating factors for constipation (Q12B). Among the most frequently evaluated exacerbants were comorbidities (38\%), concurrent medications $(32 \%)$, neurological problems $(15 \%)$, and pain on defecation (13\%).

In case of diagnosis of OIC, the most frequently used treatments were: a combination of laxatives (35\% of respondents), a PAMORA plus a laxative (28\%), a PAMORA monotherapy (19\%) or a non-pharmacological approach (14\%) (Q13A). PAMORAs' prescription levels were higher among palliative medicine specialists, anesthesiologists/pain therapists and oncologists, but we also detected rising levels among psychiatrists.

In case of OEC, the most frequently used therapy is PAMORA plus laxative $(37 \%)$ or a 
combination of laxatives (34\%) (Q13B). Nonpharmacological approaches $(14 \%)$ or PAMORA monotherapy $(13 \%)$ were prescribed in fewer cases.

Beyond dietary and lifestyle measures, 49\% of respondents indicated a preference for the osmotic laxative macrogol as prophylaxis, followed by a combination of macrogol and a stimulant laxative (e.g., senna or bisacodyl) as first-line therapy for the management of OIC (Q7). The second most common response was represented by evacuating enemas or glycerin (16\%), followed by senna or bisacodyl (12\%), and "other" (7\%). In open comments, some explanations of "other" responses specified the use of PAMORAs. Albeit with some differences, the strategies of first-line treatments for OIC show similar trends to those reported by the same specialists in the previous survey [17].

When asked in what percentage of patients receiving laxative treatment for OIC they prescribed PAMORAs, the respondents who indicated they never prescribed them were less than the past year (40\% vs $61 \%$ ), whereas those who used them in up to $10 \%$ of cases were similar (22 vs. $21 \%)(\mathrm{Q} 8)$. The frequency of PAMORAs' prescription increased, with $38 \%$ of respondents prescribing these second-line treatments in more than $10 \%$ of their cases: specifically, $18 \%$ used them in $10-30 \%$ of cases, $9 \%$ in $30-50 \%$ of cases and $11 \%$ in more than $50 \%$ of cases (Fig. 4A). While in the previous survey [17] 85\% of psychiatrists, including specialists in addiction medicine, never used PAMORAs, the updated data showed that almost half of them started using these mechanism-based pharmaceuticals. We highlight that most psychiatrists were specialists in addiction medicine and their patients were in treatment with opioids. A moderate increase in the use of PAMORAs was also found among general practitioners, with $72 \%$ of them never using these medications in 2020 reduced to $47 \%$ in 2021 .

During the past year, $46 \%$ of all the respondents modified their clinical practice in managing OIC by increasing the use of PAMORAs (Q9) (Fig. 4C). The higher percentages were observed among anesthesiologists/pain therapists (65\%) and oncologists
(60\%), who were the most likely to use PAMORAs even in the previous survey.

\section{Quality of Life and Therapy Modification}

Similar to our previous data, the updated survey confirmed that OIC and the lack of its management could negatively influence the QoL of patients to some extent. Indeed, $86 \%$ of respondents acknowledged that not managing OIC could significantly (56\%) or very significantly (30\%) impact the well-being and QoL of patients (Q10). A minority of respondents (14\%) indicated that it could affect QoL only to a limited degree.

Moreover, we detected an increase (from 55 to $70 \%$ of respondents) in the proportion of specialists who indicated that OIC can negatively influence adherence to analgesic regimens or opioid substitution therapy. Eighteen percent thought it could happen, but it depended on the patient, whereas $11 \%$ indicated that compliance with opioid therapy is not generally affected by OIC $(\mathrm{Q} 11 \mathrm{~A})$. Among the subjects who agreed that opioid-induced constipation can negatively influence adherence to opioid therapy (Q11B), 44\% indicated that they would not apply any modifications to ongoing treatment. The remaining 56\% specified that they would make changes to improve adherence, such as reduction of opioid dose and addition of a coadjuvant (39\%), reduction of opioid dosage $(12 \%)$, or switch to a weaker opioid (6\%).

\section{Patient Information}

Sixty-six percent of respondents indicated that patients were always provided information about the possible emergence of constipation and indications for its prevention/management before the initiation of treatment with an opioid and via an informative brochure (Q5A). Twenty-eight percent provided this information only upon request, at the initial onset of alterations in GI function (19\%), or even later, only when serious constipation symptoms emerge, to avoid alarming the patient needlessly (9\%) (Fig. 5A). 


\section{A In what percentage of patients receiving laxative treatment for OIC, do you prescribe a PAMORA?}

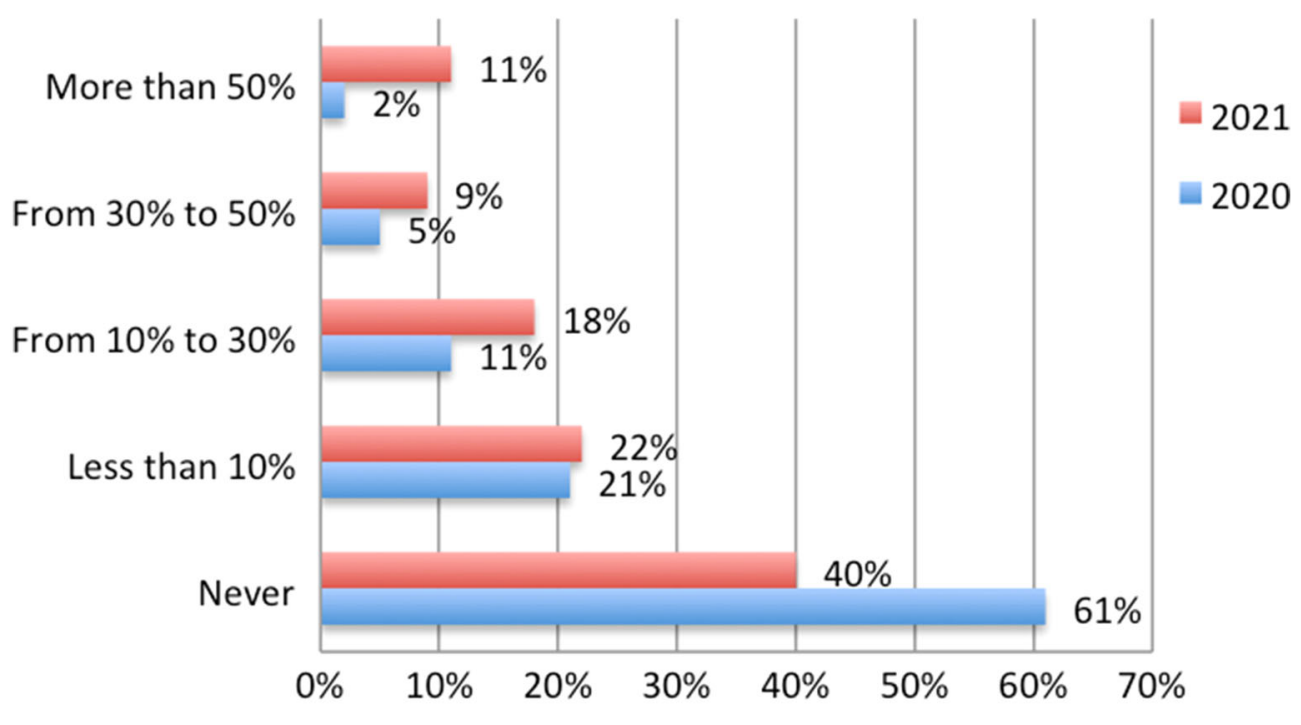

\section{B In the past year did you modify your clinical practice in patients treated for OIC, increasing the use of PAMORAs?}



Yes, I increased the use of PAMORAs

No, I did not increase the use of PAMORAs

Fig. 4 OIC treatment's strategies. A PAMORA prescriptions. B Increase in the use of PAMORAs in the past year

When asked if during the past year they provided more information to opioid-treated patients regarding the onset of potential constipation and its prevention/management, $40 \%$ of respondents answered that they provided indications to all opioid-treated patients and $25 \%$ affirmed they gave such information at the onset of first symptoms (Q5B). Thirty-two percent did not modify their practice of providing adequate advice about OIC since the start of opioid treatment (Fig. 5B), confirming a good attention of our cohort to this aspect.

\section{DISCUSSION}

Opioid-induced constipation is the most common side effect of opioid therapy that negatively affects patients' QoL and compliance with treatment. From a previous survey among Italian healthcare professionals managing patients upon opioids, we detected high levels of interest in OIC, although with important differences in the education and management of this condition across physician specialties [17]. In the present version of the survey, we 


\section{A In clinical practice, in a patient receiving treatment with an opioid agonist, information about the possible emergence of constipation symptoms and indications for its prevention/management:}

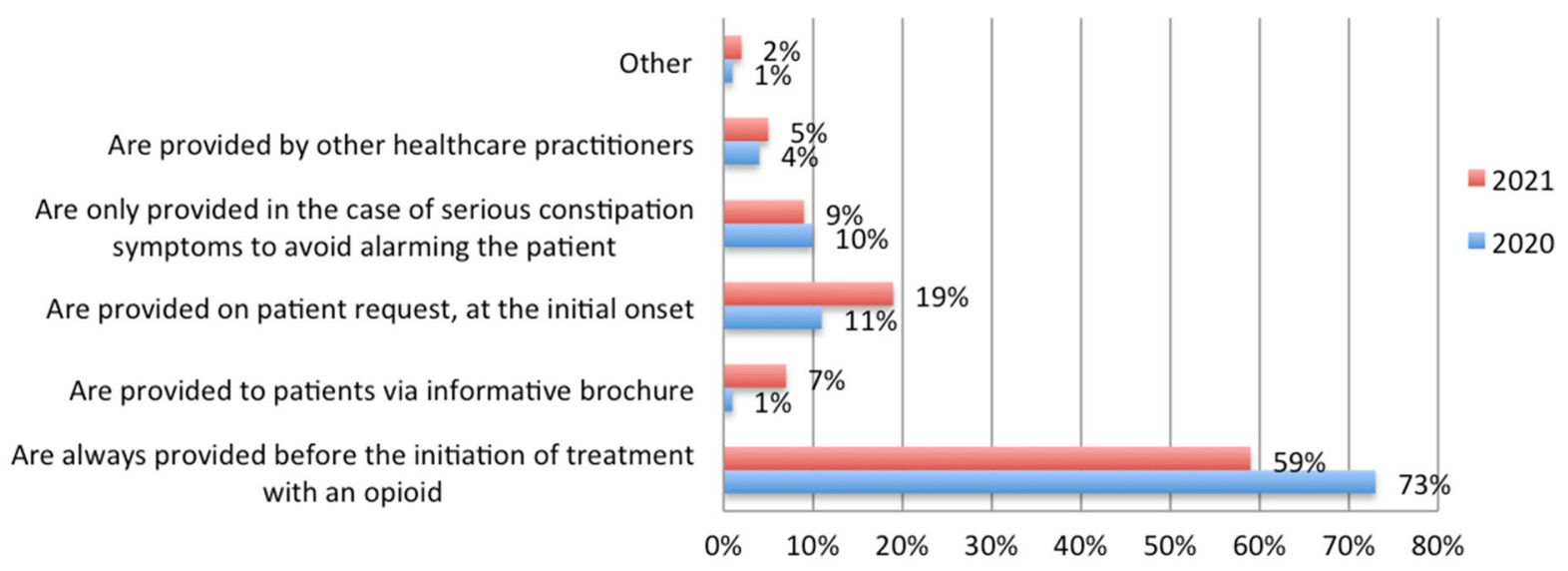
B In the past year, did you provide more information to opioid-treated patients regarding the onset of potential constipation and its prevention/management?
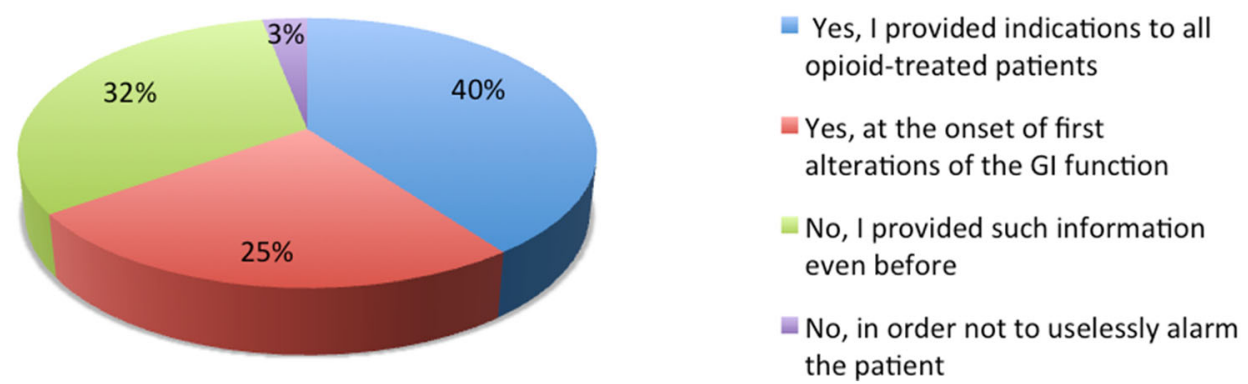

Fig. 5 Patient information. A When it is provided. B Progress in patient information

found a high and substantially unaltered interest in the topic compared to the previous year, still with some differences across disciplines. Various degrees of interest may correlate to either the number of patients with OIC seen by every specialist, or the knowledge they have about the condition.

Consistent with this second hypothesis, we detected only a very slight increase in the proportion of physicians that feel satisfied with their education on OIC management, and we observed that there is still a broad group of professionals requiring further formation on this topic. Hence, we underline the need to enact educational initiatives and campaigns to raise awareness among those specialists. In this regard, standardization of prevention, diagnosis, and treatment is sought to make all the professionals aware of the available diagnostic tools and treatment options [18].

Indeed, we reported that most of the respondents evaluated intestinal function at regular intervals, especially in patients under long-term opioid therapy, and we detected a general implementation in the assessment of intestinal function. We also observed an increase in the use of specific scales and questionnaires to assess constipation, even though the percentage of respondents who use Rome IV as diagnostic criteria is still moderate and most of respondents still rely on patient self-reports. While patient diaries could be useful to some 
extent, they are subject to patients' and physicians' bias, especially in patients with bowel obsessive syndrome or compulsion disorders related to defecation [19], and in those receiving substitution therapy [9]. In both of our surveys, we detected a significantly different approach to OIC by psychiatrists, when compared to all the other specialists. Undoubtedly, the different prescription indication used by psychiatrists (maintenance therapy versus analgesia) could have an influence on the way they perceive, assess and treat OIC. However, this hypothesis has not been tested, but a deeper understanding of the intrinsic causes of psychiatrist different approach to OIC could be an interesting topic of future research to better manage this condition even in patients suffering from opioid use disorders.

The present survey confirmed that the lack of management of OIC could have a negative impact on patients' QoL and compliance with opioid therapy. Previous research highlighted that severe adverse effects are often the reason patients discontinue opioid analgesic treatment or opioid substitution therapy, which naturally results in suboptimal pain management [20]. In order to augment the adherence to opioid therapy, a still high proportion of the surveyed professionals were willing to reduce opioid dose and/or add a coadjuvant or switch to a weaker opioid. However, this may have implications on the efficacy of the analgesic regimen, ultimately invalidating patients' QoL $[9,21]$. Therefore, it must be stressed that a better management of OIC should be based on an appropriate treatment for constipation rather than putting analgesia at risk $[16,18]$.

Besides the need for education throughout healthcare professionals, the original survey also identified the importance of developing awareness campaigns for OIC targeted to patients on long-term opioid therapy [17]. Indeed, during the past year we detected an increase in the information provided to patients about the potential onset of constipation, its prevention, and management. Nonetheless, there are still professionals that decide to wait for the first symptoms or do not give any indications because they do not want to alarm their patients. A multinational survey comprising five European countries indicated that almost $60 \%$ of physicians failed to adequately counsel their patients about constipation as a major adverse event associated with the use of opioids [22]. This may depend on poor communication between healthcare professionals and patients, which in turn could hamper effective management of OIC. Studies in a variety of chronic diseases have demonstrated that patients who have a better knowledge of their disease and are more empowered often have improved health outcomes and use better healthcare resources [23]. Healthcare professionals should timely communicate appropriate information about OIC and proactively raise the topic rather than wait for the patient to initiate the discussion [24]. As a panel of experts recently emphasized, there is a need to use strategies to better inform patients about their condition and the pros and cons of the treatment, in order to make them collaborative participants of their own care [18].

In terms of diagnosis, the current survey revealed an increase in the use of Rome IV criteria that are now considered the gold standard for OIC diagnosis [22, 25]. Especially for patients upon chronic opioid treatment, the proportion of specialists that used specific scales to diagnose OIC almost doubled compared to the previous year. Nonetheless, the implementation in the use of standardized scales was mild, and still limited to few specialists. Moreover, a lack of experience in the use of such scales is still evident, therefore educational campaigns should also be purposed to training health professionals on how to apply these scales in clinical practice. Considering that Rome IV criteria are still not used in the clinical practice of most of respondents, we highlight a clear need of spreading culture on their implementation.

Another important aspect for the differential diagnosis is the recognition of opioid-exacerbated constipation (OEC). Distinguish between OEC and OIC is not merely a semantic sophism, but a correct diagnosis can change the therapeutic approach and optimize the use of drugs. Unfortunately, epidemiologic data on the proportion of prevalence between OEC and OIC are not available yet. Some authors considered that, as $10-20 \%$ of the population suffers from 
constipation at baseline, it should be expected a similar proportion of individuals who will experience constipation as an exacerbation of their baseline condition (leading to the diagnosis of OEC) [26].

Our survey confirmed that a critical point is to evaluate and specifically address exacerbating factors for constipation, in particular comorbid conditions and concomitant pharmacological therapies, but also neurological problems and pain on defecation. Prior to initiating an opioid therapy, an accurate anamnesis with particular focus on the baseline bowel habit and a detailed drug history is necessary for a timely recognition of exacerbating factors and, where possible, a modification of concurrent medications.

Since OIC symptoms are difficult to tolerate and unlikely to resolve on their own with time, specific prevention and treatment are needed. Dietary and lifestyle measures are certainly important, but the guidelines issued by several medical associations [11-13, 15, 27], the recent European expert consensus statement [16] and two Italian multidisciplinary expert groups $[18,28]$ recommend prophylaxis and first-line treatment of OIC with standard laxatives, either osmotic agents (macrogol) or stimulants (senna, bisacodyl). In accordance with these guidelines and similarly to our previous survey, the majority of respondents preferred a combination of macrogol and another laxative as firstline treatment for OIC. However, currently there are no randomized controlled trials that support the use of standard laxatives for the treatment of OIC and most of them have limited efficacy [29], making it necessary to search for new strategies.

One promising option is represented by PAMORAs that specifically target the underlying cause of this condition without affecting centrally mediated pain relief. The safety and efficacy of three main PAMORAs-methylnaltrexone, naldemedine and naloxegol-have been documented in a variety of studies, including large randomized controlled trials $[30,31]$. Despite PAMORAs are recommended as second-line treatment for OIC [12, 16, 32], our original survey demonstrated an underutilization of these targeted agents within Italian specialists, with the exception of oncologists and anesthesiologists/pain therapists [17]. The current study demonstrated an increase in the use of PAMORAs, with nearly half of respondents having modified their clinical practice during the past year by introducing these pharmacological agents in the treatment of patients with OIC. This implementation may have been influenced by the regulatory and reimbursement changes recently introduced in Italy by AIFA with the modification of nota 90.

Although still a relevant proportion of physicians managing OIC patients never prescribed PAMORAs, nearly two-thirds of respondents declared to utilize them as a second-line therapy in some of their patients. We noticed there is still a fragmented use of PAMORAs across clinical specialties, with pain therapists/ anesthesiologists, oncologists, and palliative medicine specialists being the most virtuous, and other professionals being more resistant to their administration.

The present study had some limitations. First, an adequate comparison with the previous survey was hampered by the low adherence of physicians, as only half of the respondents of the original survey accepted the invitation to the updated inquiry. Despite an extension of the duration, the poor responsiveness may have depended at least in part on the chosen time period, just before holidays in Italy.

Another limitation is that not all the medical specialties maintained the same proportions as the previous study, thus limiting a detailed comparison of aggregated physician specialties. Moreover, we point out that surveys are inherently subjected to intrinsic confounder factors, such as selection and recall biases. Effects of time and memory factors should also have been taken in consideration as potential limitations in evaluating the reliability of answers.

\section{CONCLUSIONS}

Our data demonstrate that some implementation in the management of OIC was observed among our cohort of healthcare providers during the past year. Thirty-three percent of respondents implemented their routine assessment of pain. Moreover, one-third of the 
centers where these specialists worked implemented the assessment of intestinal function of opioid-treated patients, with a mild but significant increase in the use of standardized scales, especially in patients under long-term opioid therapy. The differential diagnosis between OIC and OEC was considered important by the whole cohort of experts and the majority of the surveyed physicians followed the guidelines for first-line treatment of OIC. Importantly, the modification of nota 90 from AIFA prompted a broader use of PAMORAs as demonstrated by a significant implementation in their prescription. Despite these positive trends, we still notice a partial awareness about OIC, especially across some specialties, hence we highlight the need for further education and standardization of specific diagnostic and treatment options and a better engagement of patients in the management of this condition.

\section{ACKNOWLEDGEMENTS}

Funding. An unconditional grant for this study and the journal's Rapid Service Fee were provided by Shionogi Srl, Rome, Italy.

Medical Writing, Editorial, and Other Assistance. The authors would like to thank Cristina Colombelli, PhD, freelance medical writer for draft preparation and Cinzia Filoso, PM of Sanitanova Srl, for editorial assistance. This assistance was funded by Shionogi Srl, Rome, Italy.

Authorship. All named authors meet the International Committee of Medical Journal Editors (ICMJE) criteria for authorship for this article, take responsibility for the integrity of the work as a whole, and have given their approval for this version to be published.

Author Contributions. All authors contributed equally to the manuscript. All of them made a significant contribution to the work reported, whether that is in the conception, study design, execution, acquisition of data, analysis and interpretation, or in all these areas; took part in drafting, revising or critically reviewing the article; gave final approval of the version to be published; have agreed on the journal to which the article has been submitted; and agree to be accountable for all aspects of the work.

Disclosures. Domenico Alvaro has received research grants and consultant fees from Intercept Pharma, Molteni Farmaceutici, Shionogi, Vesta and is a consultant for Aboca. Flaminia Coluzzi is speaker and consultant for Angelini, Grunenthal, Malesci, Molteni, Pfizer, Shionogi. Walter Gianni, Fabio Lugoboni, Franco Mariangeli, Giuseppe Massazza, Carmine Pinto, and Giustino Varrassi have received research grants and other funding from Molteni and Shionogi. Giustino Varrassi is also a member of the Advisor Boards of Abbott, Dompé, Malesci, Menarini International, Molteni, Mundipharma, Shionogi. He is also Member of the Speakers' Bureau of Berlin-Chemie, Dompé, MAP, Menarini International, MCAC, Molteni, Takeda. He has received funds for research by Dompé, Fondazione Maugeri, and Pfizer, and he is Editor-inChief of Pain and Therapy.

Compliance with Ethics Guidelines. This article does not contain any studies with human or animals, and it is not subject to approval by an ethical committee, according to the national law (Italian Law 11 January 2018 and Italian Legislative decree 14 May 2019, nr. $52)$. The participation in the survey was completely anonymous and voluntary, and all clinicians have indicated their willingness to voluntarily take part in the survey. A survey tool generates a numeric code for each participant which prevents the authors from identifying him/her and results are reported in aggregated form. Survey responses were anonymized and handled via remote dispersed geographical participation. The invitation to the survey declared the content of the study and gave the opportunity to join to the project or deny the involvement, or terminate the participation at any time without penalty. By accepting our invitations each participant expresses the informed consent according to the legislation for the protection of privacy (Legislative Decree 
196/2003-European Regulation (EU) 679/2016). All the physicians involved in the survey consented to have their responses used in a medical publication.

Data Availability. Data sharing is not applicable to this article as no datasets were generated or analyzed during the current study.

Open Access. This article is licensed under a Creative Commons Attribution-NonCommercial 4.0 International License, which permits any non-commercial use, sharing, adaptation, distribution and reproduction in any medium or format, as long as you give appropriate credit to the original author(s) and the source, provide a link to the Creative Commons licence, and indicate if changes were made. The images or other third party material in this article are included in the article's Creative Commons licence, unless indicated otherwise in a credit line to the material. If material is not included in the article's Creative Commons licence and your intended use is not permitted by statutory regulation or exceeds the permitted use, you will need to obtain permission directly from the copyright holder. To view a copy of this licence, visit http://creativecommons.org/licenses/by$\mathrm{nc} / 4.0 /$.

\section{REFERENCES}

1. Benyamin R, Trescot AM, Datta $S$, et al. Opioid complications and side effect. Pain Physician. 2008;11(2 Suppl):S105-20.

2. Camilleri M, Drossman DA, Becker G, Webster LR, Davies AN, Mawe GM. Emerging treatments in neurogastroenterology: a multidisciplinary working group consensus statement on opioid-induced constipation. Neurogastroenterol Motil. 2014;26(10):1386-95.

3. Bell T, Annunziata K, Leslie JB. Opioid-induced constipation negatively impacts pain management, productivity, and health-related quality of life: findings from the National Health and Wellness Survey. J Opioid Manag. 2009;5(3):137-44.

4. Veiga DR, Mendonça L, Sampaio R, Lopes JC, Azevedo LF. Incidence and health related quality of life of opioid-induced constipation in chronic noncancer pain patients: a prospective multicentre cohort study. Pain Res Treat. 2018;2018:5704627.

5. Varrassi G, Banerji V, Gianni W, Marinangeli F, Pinto C. Impact and consequences of opioid-induced constipation: a survey of patients. Pain Ther. 2021;10(2):1139-53.

6. Glare P, Walsh D, Sheehan D. The adverse effects of morphine: a prospective survey of common symptoms during repeated dosing for chronic cancer pain. Am J Hosp Palliat Care. 2006;23(3):229-35.

7. Drewes A, Munkholm P, Simrén M, et al. Definition, diagnosis and treatment strategies for opioidinduced bowel dysfunction-recommendations of the Nordic Working Group. Scand J Pain. 2016;11: 111-22.

8. Tuteja AK, Biskupiak J, Stoddard GJ, Lipman AG. Opioid-induced bowel disorders and narcotic bowel syndrome in patients with chronic non-cancer pain. Neurogastroenterol Motil. 2010;22(4):424-30 (e96).

9. Troberg K, Håkansson A, Dahlman D. Self-rated physical health and unmet healthcare needs among Swedish patients in opioid substitution treatment. J Addict. 2019;15:7942145.

10. Poulsen JL, Nilsson M, Brock C, Sandberg TH, Krogh $\mathrm{K}$, Drewes AM. The impact of opioid treatment on regional gastrointestinal transit. J Neurogastroenterol Motil. 2016;22(2):282-91.

11. Caraceni A, Hanks G, Kaasa S, et al. Use of opioid analgesics in the treatment of cancer pain: evidence-based recommendations from the EAPC. Lancet Oncol. 2012;13(2):e58-68.

12. Kampman K, Jarvis M. American Society of Addiction Medicine (ASAM) National Practice Guideline for the Use of Medications in the Treatment of Addiction Involving Opioid Use. J Addict Med. 2015;9(5):358-67.

13. Crockett S, Greer KB, Sultan S. Opioid-Induced Constipation (OIC) Guideline. Gastroenterology. 2019;156(1):228.

14. Crockett SD, Greer KB, Heidelbaugh JJ, et al. American Gastroenterological Association Institute Guideline on the medical management of opioidinduced constipation. Gastroenterology. 2019;156(1):218-26.

15. Müller-Lissner S, Bassotti G, Coffin B, et al. Opioidinduced constipation and bowel dysfunction: a clinical guideline. Pain Med. 2017;18(10):1837-63.

16. Farmer AD, Drewes AM, Chiarioni G, et al. Pathophysiology and management of opioid-induced 
constipation: European expert consensus statement. United European Gastroenterol J. 2019;7(1): 7-20.

17. Coluzzi F, Alvaro D, Caraceni AT, et al. Common clinical practice for opioid-induced constipation: a physician survey. J Pain Res. 2021;14:2255-64.

18. Alvaro D, Caraceni AT, Coluzzi F, et al. What to do and what not to do in the management of opioidinduced constipation: a choosing wisely report. Pain Ther. 2020;9(2):657-67.

19. Cosci F. "Bowel obsession syndrome" in a patient with chronic constipation. Gen Hosp Psychiatry. 2013;35(4):451.e1-3.

20. Thorpe DM. Management of opioid-induced constipation. Curr Pain Headache Rep. 2001;5(3): 237-40.

21. Dueñas M, Ojeda B, Salazar A, Mico JA, Failde I. A review of chronic pain impact on patients, their social environment and the health care system. J Pain Res. 2016;9:457-67.

22. Andresen V, Banerji V, Hall G, Lass A, Emmanuel A. The patient burden of opioid-induced constipation: new insights from a large, multinational survey in five European countries. United European Gastroenterol J. 2018;6(8):1254-66.

23. Simmons LA, Wolever RQ, Bechard EM, Snyderman $\mathrm{R}$. Patient engagement as a risk factor in personalized health care: a systematic review of the literature on chronic disease. Genome Med. 2014;6(2): 16.

24. Epstein RS, Teagarden JR, Cimen A, Sostek M, Salimi T. When people with opioid-induced constipation speak: a patient survey. Adv Ther. 2017;34(3):725-31.
25. Simren M, Palsson OS, Whitehead WE. Update on Rome IV criteria for colorectal disorders: implications for clinical practice. Curr Gastroenterol Rep. 2017;19(4):15.

26. Brenner DM, Stern E, Cash BD. Opioid-related constipation in patients with non-cancer pain syndromes: a review of evidence-based therapies and justification for a change in nomenclature. Curr Gastroenterol Rep. 2017;19(3):12.

27. Larkin PJ, Cherny NI, La Carpia D, et al. Diagnosis, assessment and management of constipation in advanced cancer: ESMO Clinical Practice Guidelines. Ann Oncol. 2018;29(Suppl 4):iv111-25.

28. Rossi M, Casale G, Badiali D, et al. Opioid-induced bowel dysfunction: suggestions from a multidisciplinary expert Board. Support Care Cancer. 2019;27(11):4083-90.

29. Emmanuel A, Johnson M, McSkimming P, Dickerson $\mathrm{S}$. Laxatives do not improve symptoms of opioid-induced constipation: results of a patient survey. Pain Med. 2017;18(10):1932-40.

30. Rekatsina M, Paladini A, Drewes AM, et al. Efficacy and Safety of Peripherally Acting $\mu$-Opioid Receptor Antagonist (PAMORAs) for the management of patients with opioid-induced constipation: a systematic review. Cureus. 2021;13(7):e16201.

31. Nee J, Zakari M, Sugarman MA, et al. Efficacy of treatments for opioid-induced constipation: systematic review and meta-analysis. Clin Gastroenterol Hepatol. 2018;16(10):1569-1584.e2.

32. Pergolizzi JV Jr, Christo PJ, LeQuang JA, Magnusson $P$. The use of peripheral $\mu$-opioid receptor antagonists (PAMORA) in the management of opioid-induced constipation: an update on their efficacy and safety. Drug Des Devel Ther. 2020;14:1009-25. 